\title{
Thyroid dysfunction during pregnancy and in the first postpartum year in women with diabetes mellitus type 1
}

\author{
P Robert J Gallas, Ronald P Stolk ${ }^{1}$, Karel Bakker ${ }^{2}$, Erik Endert and Wilmar M Wiersinga \\ Department of Endocrinology and Metabolism, Academic Medical Centre, Meibergdreef 9, 1105 AZ Amsterdam, The Netherlands, \\ ${ }^{1}$ University Medical Centre Utrecht, Heidelberglaan 100, 3584 CX Utrecht, The Netherlands and ${ }^{2}$ Spaarne Ziekenhuis Heemstede, \\ Heemsteedse Dreef 90, 2102 KN Heemstede, The Netherlands \\ (Correspondence should be addressed to P R J Gallas, Griffensteijnseplein 16, 3703 BG Zeist, The Netherlands; Email: rgallas@medweb.nl)
}

(P R J Gallas is now at Diakonessenhuis Zeist, Prof. Lorentzlaan 76, 3707 HL Zeist, The Netherlands)

\begin{abstract}
Background: The prevalence of thyroid dysfunction in pregnancy and in the first postpartum year (postpartum thyroid dysfunction (PPTD)) in women with diabetes mellitus type 1 (DM1) is known to be higher than in the general population. To assess prevalence, incidence and risk factors in The Netherlands we performed a prospective cohort study.

Design: From 1994 to 1998, 126 women with DM1 from eight Dutch clinics were included. TSH, free thyroxine, free tri-iodothyronine and anti-thyroid peroxidase antibodies (TPO-ab) were measured prepregnancy, in the first and last trimester of pregnancy and at 1.5, 3, 6, 9 and 12 months after delivery. Results: Eighty-two women completed the study. Thyroid dysfunction during pregnancy was observed in $22.5 \%$ (first trimester) and $18.4 \%$ (third trimester), and mostly consisted of subclinical hypothyroidism. Baseline characteristics of women with thyroid dysfunction in pregnancy did not differ from those without thyroid dysfunction. Overt PPTD was seen in $15.9 \%$. Incidence of PPTD was $10 \%$. Patients with PPTD were slightly older than those without PPTD and the prevalence of TPO-ab was higher in these women.

Conclusion: In women with DM1 the prevalence of thyroid dysfunction during pregnancy and overt PPTD is 3-fold higher than in the general Dutch population. Risk factors are age and TPO-ab. Given the possible impact on psychomotor development of the offspring and on well-being of the mother these data suggest there is a case for screening (pre-)pregnant women with DM1 for TSH and TPO-ab.
\end{abstract}

European Journal of Endocrinology 147 443-451

\section{Introduction}

Diabetes mellitus type 1 (DM1) is associated with other auto-immune disorders, in particular auto-immune thyroid disease $(1,2)$. Women with DM1 are found to have anti-thyroid peroxidase antibodies (TPO-ab) in a higher proportion (up to 20\%) than the background population (3), and their prevalence of auto-immune hypothyroidism is also higher $(4,5)$.

Pregnancy and the postpartum period tend to increase the incidence and level of thyroid dysfunction (TD) in women with auto-immune thyroid disease (AITD) $(6,7)$. The implications for women with DM1 are partly known. In pregnancy, hormonal demands on the thyroid are increased by several mechanisms (8). As the functional capacity of the thyroid in AITD is impaired, and AITD is more prevalent in women with DM1 one might expect an increasing prevalence of TD in the diabetic pregnancy. However, information on thyroid (dys)function in pregnant women with DM1 is scarce; TD varies from 0 to $16 \%$ (predominantly subclinical hypothyroidism) $(9,10)$.

When TPO-ab are present in pregnancy there is an increased risk of development of postpartum TD (PPTD) (11). PPTD is caused by a destructive autoimmune thyroiditis ('postpartum thyroiditis') mediated by rebound of both cellular and humoral immunity in the postpartum period $(7,12)$. The clinical presentation varies from hyperthyroidism, hypothyroidism or both and is associated with significant symptomatology, including an increased incidence of minor to moderate depression $(12,13)$.

Although PPTD is often transient, 23\% of the women will have definitive hypothyroidism after 3 years (14). Owing to the higher prevalence of AITD, the risk of a woman with DM1 to develop PPTD is three times 
higher than the risk for normal women, according to studies in Denmark (9), Canada (15) and the USA (16). So far no study has been done starting the follow-up during the pre-pregnant period. We performed a multicentre prospective study to assess the incidence, prevalence and determinants of TD in a cohort of women with DM1 from pre-pregnancy until 1 year postpartum.

\section{Subjects and methods}

\section{Patients}

From 1994 to 1998, 126 women with DM1 from eight outpatient clinics throughout the Netherlands (Academisch Medisch Centrum Amsterdam, Academisch Ziekenhuis van de Vrije Universiteit Amsterdam, Catharina Ziekenhuis Eindhoven, Academisch Ziekenhuis Universiteit Groningen, Spaarne Ziekenhuis Heemstede, Leiden University Medical Centre, Westeinde Ziekenhuis Den Haag and Isala Klinieken lokatie Weezenlanden Zwolle) were enrolled in the study and followed prospectively. The women had to be pregnant (but not longer than 14 weeks from the first day of the last period) or desiring to become pregnant. Excluded were all women with a history of thyroid disease and those using immunosuppressive medication. Women who experienced a spontaneous miscarriage were asked to continue the study. In the case of renewed pregnancy in the first year postpartum the follow-up was restarted from the last pregnancy onwards.

The women gave written informed consent to the study, which was approved by all local ethical committees.

\section{Methods}

The women were seen before pregnancy if possible, twice in their pregnancy (in the first and third trimester) and consecutively 6 weeks and 3, 6, 9 and 12 months after delivery. At each visit blood was drawn to measure thyrotrophin (TSH), free thyroxine $\left(\mathrm{FT}_{4}\right)$, free tri-iodothyronine $\left(\mathrm{FT}_{3}\right)$, TPO-ab, TSH-receptor binding inhibiting immunoglobulins (TBII) and glycosylated haemoglobin (HbA1c).

\section{Thyroid function tests}

$\mathrm{FT}_{4}$ was measured by a fluoroimmunoassay (Delfia; Wallac Oy, Turku, Finland); reference range 8.7-19.6 pmol/l, as defined for non-pregnant women aged 20-40 years originating from the region Kempenland in the southeast Netherlands.

$\mathrm{FT}_{3}$ fluoroimmunoassay (Delfia; Wallac Oy) was performed in the case of a decreased TSH but a normal $\mathrm{FT}_{4}$ to detect a possible $\mathrm{T}_{3}$ toxicosis; reference values $4.6-7.8 \mathrm{pmol} / \mathrm{l}$.
TSH was determined using a time-resolved fluoroimmunoassay (Delfia hTSH Ultra; Wallac Oy); reference range $0.15-2.0 \mathrm{mU} / \mathrm{l}$, as defined for 225 non-pregnant women aged 20-40 years originating from the region Kempenland in the southeast Netherlands, using the International Federation of Clinical Chemistry recommendations for defining reference values (17).

TPO-ab were assessed by a luminescence immunoassay (Lumitest anti-TPO; Brahms Diagnostica GMBH, Berlin, Germany); a concentration of $>100 \mathrm{kU} / \mathrm{l}$ was defined as 'positive'.

TBII was measured using a radio-receptor assay (TRAK-assay; Brahms); reference range <9 U/l antibody-negative, 9-14 U/l borderline, $>14 \mathrm{U} / \mathrm{l}$ positive.

$\mathrm{HbA1c}$ measurements were performed locally with various methods and corrected afterwards using correction factors produced by an external quality control programme, according to the Diabetes Control and Complications Trial standard (18).

\section{Definitions}

TD and PPTD were defined as the presence of an abnormal TSH $(\mathrm{TSH}<0.15 \mathrm{mU} / \mathrm{l}$ or $>2.0 \mathrm{mU} / \mathrm{l})$. Further classification in overt or subclinical TD (or PPTD) was performed according to the results of $\mathrm{FT}_{4}$ and $\mathrm{FT}_{3}$. $\mathrm{FT}_{3}$ was measured only in the case of an abnormal TSH and a normal $\mathrm{FT}_{4}$, to detect a possible $\mathrm{T}_{3}$ toxicosis: low TSH, high $\mathrm{FT}_{4}$ or $\mathrm{FT}_{3}$ - overt hyperthyroidism; low $\mathrm{TSH}$, normal $\mathrm{FT}_{4}$ and $\mathrm{FT}_{3}$ - subclinical hyperthyroidism; high TSH and low $\mathrm{FT}_{4}$ - overt hypothyroidism; high $\mathrm{TSH}$ and normal $\mathrm{FT}_{4}$ - subclinical hypothyroidism.

\section{Analysis}

Statistical analysis was performed using SPSS version 9.0. Median and interquartile ranges are given for thyroid function at different time points. Differences between subgroups were analysed using $t$-tests for continuous variables, Mann-Whitney and Fisher exact tests for categorical variables.

\section{Results}

From the women who were enrolled in the study, 15 did not get pregnant during the study period. Another 29 did not attend at least two time points in the postpartum period. Three women experienced a miscarriage. All three completed the follow-up period (until one year after the miscarriage). Two women got pregnant again in the first year postpartum; the follow-up period was restarted from the last measurement before their second pregnancy.

The study population finally consisted of 82 women (81 Caucasian, 1 black). Thirty-six women were included before gestation, the other 46 in the first 12 
weeks of pregnancy. During pregnancy 38 women $(46.3 \%)$ were treated with continuous s.c. insulin infusion, $43(52.4 \%)$ with three injections of shortacting and one injection of long-acting insulin a day and one woman (1.2\%) injected a mix of short-acting and long-acting insulin twice a day. Other characteristics of the study population are presented in Table 1 .

\section{Thyroid auto-immunity and thyroid function}

From the 36 women who were included before pregnancy, 5 of 36 (13.9\%) were TPO-ab-positive. All women positive for TPO-ab before pregnancy were also positive in the first trimester. The percentage of women with TPO-ab increased to 17 of $82(20.7 \%)$ in the first trimester. Clinical characteristics of the women with and without TPO-ab pre-pregnancy or in the first trimester did not differ (Table 1). The point prevalence of TPO-ab positivity decreased to $12.1 \%$ at 6 weeks postpartum, and then rose to a maximum of $33.8 \%$ at 6 months postpartum (Fig. 1a).

In Fig. $1 b$ the course of the TPO-ab titres of the women who were positive at each time point concerned is depicted. Antibody titres decline during pregnancy, and rise in the postpartum period with a maximum at 3 months postpartum.

During pregnancy a conspicuous decline of $\mathrm{FT}_{4}$ levels to low-normal values was seen, with immediate recurrence of normal values postpartum. TSH levels showed a slight increase within the normal range during pregnancy and in the postpartum period (Fig. 2).

In the TPO-ab-positive women, TSH was significantly higher in the first trimester of pregnancy, and at 6, 9 and 12 months after pregnancy compared with TPO-ab-negative women $\left(P<0.05\right.$, Fig. 3a). $\mathrm{FT}_{4}$ did not differ between the groups during pregnancy, but was significantly lower in the TPO-ab-positive women at 6 months postpartum (Fig. 3b). Median TSH during the whole study period in the TPO-ab-positive women was higher than in the TPO-ab-negative women (1.6 vs $1.2 \mathrm{mU} / \mathrm{l}, P<0.05)$ ). Mean $\mathrm{FT}_{4}$ did not differ between the groups (12.35 vs $12.81 \mathrm{pmol} / \mathrm{l})$. Diabetes characteristics, including duration of disease, mean $\mathrm{HbA1c}$ during the study period and presence of complications did not differ between the groups.

\section{TD}

In Fig. 4 the point prevalence of TD before, during and after pregnancy is shown. One case of $\mathrm{T}_{3}$ toxicosis in the postpartum period was found, and classified as overt hyperthyroidism.

TD before pregnancy of the women included before pregnancy one $(2.7 \%)$ had a subclinical hyperthyroidism; $10.8 \%$ of the women had a subclinical hypothyroidism. There was no overt TD.

\section{TD during pregnancy}

Prevalence TD was seen in 22 of 82 women $(26.8 \%)$ in the first and/or last trimester of pregnancy. 19 of the 82 women $(23.2 \%)$ had a subclinical or overt hypothyroidism in the first or last trimester; overt hypothyroidism was seen in 4 of $82(4.9 \%)$, subclinical hypothyroidism in 15 of $82(18.3 \%)$. There was no association between TPO-ab positivity in the first trimester with overt or subclinical hypothyroidism in pregnancy.

Subclinical hyperthyroidism was seen in 3 of 82 women $(3.7 \%)$, two in the first and one in the last trimester. Women with TD $(n=22)$ during pregnancy did not differ from those without TD $(n=60)$ with respect to age at conception, diabetes duration and complications, obstetric history, smoking habits and $\mathrm{HbA1c}$ at the start of pregnancy.

Incidence Of the 36 women included pre-pregnancy, 31 were euthyroid; 6 of these 31 women (19.4\%) developed TD during pregnancy (4 of 31 (12.9\%) subclinical hypothyroidism with a mildly elevated TSH, 2 of $31(6.5 \%)$ subclinical hyperthyroidism in the first trimester). None of these women were

Table 1 Baseline characteristics of 82 pregnant women with DM1.

\begin{tabular}{llll}
\hline & & \multicolumn{2}{c}{$\begin{array}{c}\text { TPO-ab status (pre-pregnancy } \\
\text { or in 1st trimester) }\end{array}$} \\
\cline { 2 - 4 } & All women & Positive & Negative \\
Number of women & 82 & 17 & 65 \\
Age at conception (mean (S.D.)) (years) & $30(4.2)$ & $30(4.5)$ & $30(3.9)$ \\
Body mass index at conception (mean (S.D.)) & $24.3(3.1)$ & $24.8(3.4)$ & $24.1(3.0)$ \\
Duration of diabetes at conception (mean (S.D.)) (years) & $13.6(7.8)$ & $14.3(8.4)$ & $13.4(7.7)$ \\
Women with nephropathy (\%)* & 12.2 & 11.7 & 12.3 \\
Women with retinopathy (\%) & 43.9 & 52.9 & 41.5 \\
Women with one or more previous pregnancies (\%) & $51.2(n=42)$ & $53(n=9)$ & $51(n=33)$ \\
Women with previous miscarriages (\%) & $18.3(n=15)$ & $23.5(n=4)$ & $16.9(n=11)$ \\
\hline
\end{tabular}

*Albuminuria $>300 \mathrm{mg} / 24 \mathrm{~h}$.

** Background or proliferative retinopathy. 

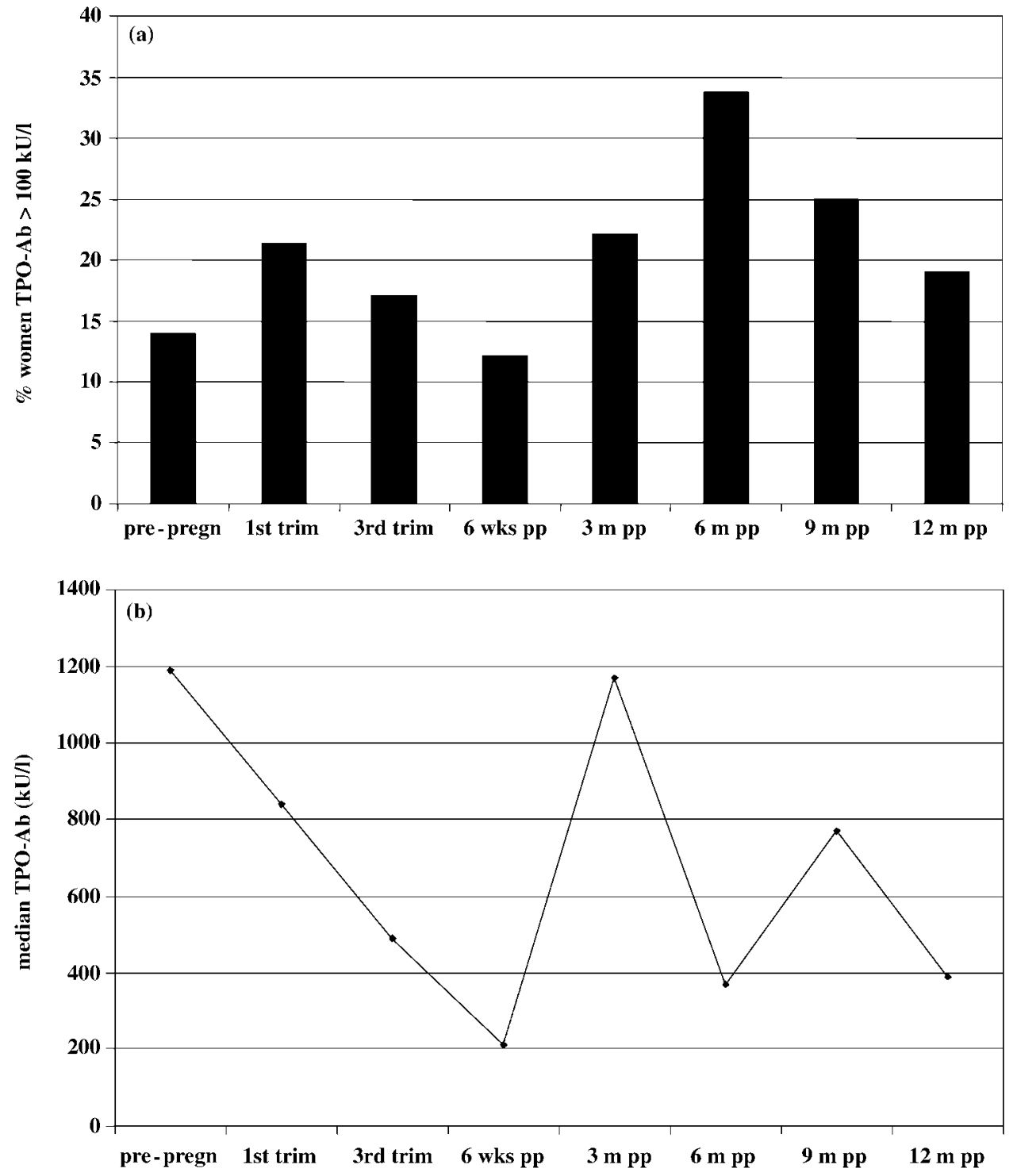

Figure 1 (a) Proportion of women with DM1 who were positive for TPO-ab at each time point. The percentage of women positive for TPO-ab decreases towards the end of pregnancy and rises with a maximum at 6 months postpartum. (b) Median TPO-ab titres in women positive for TPO-ab at each time point. TPO-ab titres show a decrease during pregnancy, rise shortly after pregnancy and then decrease towards the end of the first postpartum year. pre-pregn, pre-pregnant; trim, trimester; wks, weeks; m, months; pp, postpartum.

TPO-ab-positive. (Sub)clinical hypothyroidism during pregnancy developed in 4 of 28 women (14.3\%) with a normal TSH and no TPO-ab before pregnancy and in none of the three women with normal TSH but positive TPO-ab before pregnancy (not significant). Also when lowering the threshold for TPO-ab positivity from 100 to $50 \mathrm{kU} / \mathrm{l}$ there is no association between TPO-ab and (sub)clinical hypothyroidism in pregnancy.

\section{TD in the postpartum period}

Prevalence PPTD was prevalent in 42 of 82 women $(51 \%)$ in the first postpartum year at one or more time points (Fig. 4). Most of the women had a subclinical hypothyroidism. Overt PPTD was found in 13 of 82 women $(15.9 \%)$. Four of these 13 women had hyperthyroidism alone (one was a $\mathrm{T}_{3}$ toxicosis), hypothyroidism alone occurred in six and hyperthyroidism followed by hypothyroidism in three. Overt hypothyroidism was always transient. Two of the women were TBII-positive. One developed a persistent hyperthyroidism compatible with Graves' disease, the other one showed the classical course of postpartum thyroiditis (hyperthyroidism followed by hypothyroidism).

Seven of the 13 women (53\%) with overt PPTD already had subclinical hypothyroidism in the first 


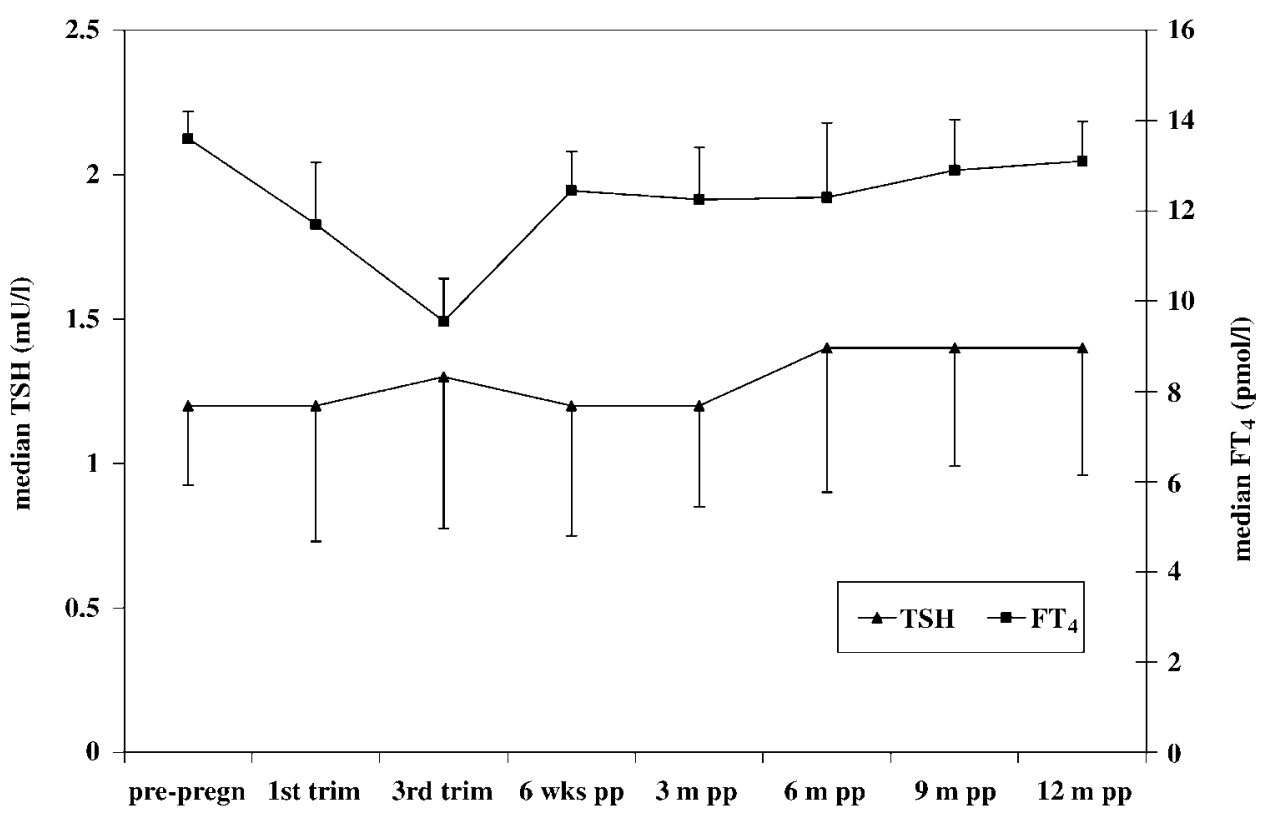

Figure $2 \mathrm{TSH}$ and $\mathrm{FT}_{4}$ (median and 25th or 75th percentile) before, during and after pregnancy. $\mathrm{FT}_{4}$ decreases during pregnancy to low-normal values, postpartum levels return to pre-pregnancy values; TSH levels show a slight increase during pregnancy and postpartum. pre-pregn, pre-pregnant; trim, trimester; wks, weeks; m, months; pp, postpartum.

trimester of pregnancy. Of these seven women, two developed overt hypothyroidism in the third trimester. Six of the 13 women with overt PPTD were euthyroid in pregnancy.

From the 14 women with a subclinical hypothyroidism in the first trimester five (35.7\%) developed overt PPTD.

None of the three women with a miscarriage developed PPTD.

Baseline characteristics of the women with and without overt PPTD are given in Table 2. The women who had overt PPTD were slightly older and 6 of 13 $(46.2 \%)$ were TPO-ab-positive in the first trimester (vs $16.4 \%$ of the women who did not have PPTD).

Incidence Of the 60 women who were euthyroid during pregnancy, 23 (38\%) developed PPTD. Overt PPTD (abnormal TSH with an abnormal $\mathrm{FT}_{4}$ or $\mathrm{FT}_{3}$ ) was seen in 6 of the $60(10 \%)$. Of these six women, three developed hyperthyroidism and one developed hypothyroidism at 3 months postpartum. The other two developed hypo- or hyperthyroidism at 6 months postpartum. Two of the six women (33.3\%) were TPO-ab-positive in the first trimester. Baseline characteristics of the women with newly developed overt PPTD did not differ from the rest of the women. Positive predictive value of TPO-ab positivity was low: 0.12 , 0.08 and $0 \%$ in the first-, last trimester and 6 weeks postpartum respectively.

From the women with normal TSH and no TPO-ab before pregnancy 14 of 28 (50\%) developed subclinical or overt PPTD vs two of three $(66.7 \%)$ from the women with a normal TSH and TPO-ab before pregnancy, which is a non-significant difference.

From the 12 women who were euthyroid in pregnancy and had TPO-ab pre-pregnancy or in the

Table 2 Baseline characteristics of women with overt PPTD vs women without overt PPTD (prevalence).

\begin{tabular}{|c|c|c|c|}
\hline & PPTD & No PPTD & Significance \\
\hline Number of women & 13 & 69 & - \\
\hline Age at conception (mean (S.D.)) (years) & $32(4.1)$ & $29.4(4.1)$ & $P<0.05$ \\
\hline Body mass index at conception (mean S.D.)) & $25.0(3.1)$ & $24.2(3.1)$ & NS \\
\hline Smoking during pregnancy (\%) & 16.7 & 16.9 & NS \\
\hline During of diabetes at conception (mean (S.D.)) (years) & $13.1(8.4)$ & $13.7(7.8)$ & NS \\
\hline TPO-ab-positive in the 1st trimester (\%) & 46.2 & 16.4 & $P<0.05$ \\
\hline TSH in 1st trimester (median $(95 \% \mathrm{Cl})(\mathrm{mU} / \mathrm{l})$ & $2.1(1.2-4.9)$ & $1.1(0.9-1.3)$ & $P<0.05$ \\
\hline $\mathrm{HbA1c}$ in pregnancy (mean (S.D.)) (\%) & $6.5(0.3)$ & $6.3(0.3)$ & NS \\
\hline HbA1c in postpartum period (mean (S.D.)) (\%) & $7.0(0.7)$ & $7.3(0.5)$ & NS \\
\hline Breastfeeding (\%) & 66.7 & 70 & NS \\
\hline
\end{tabular}



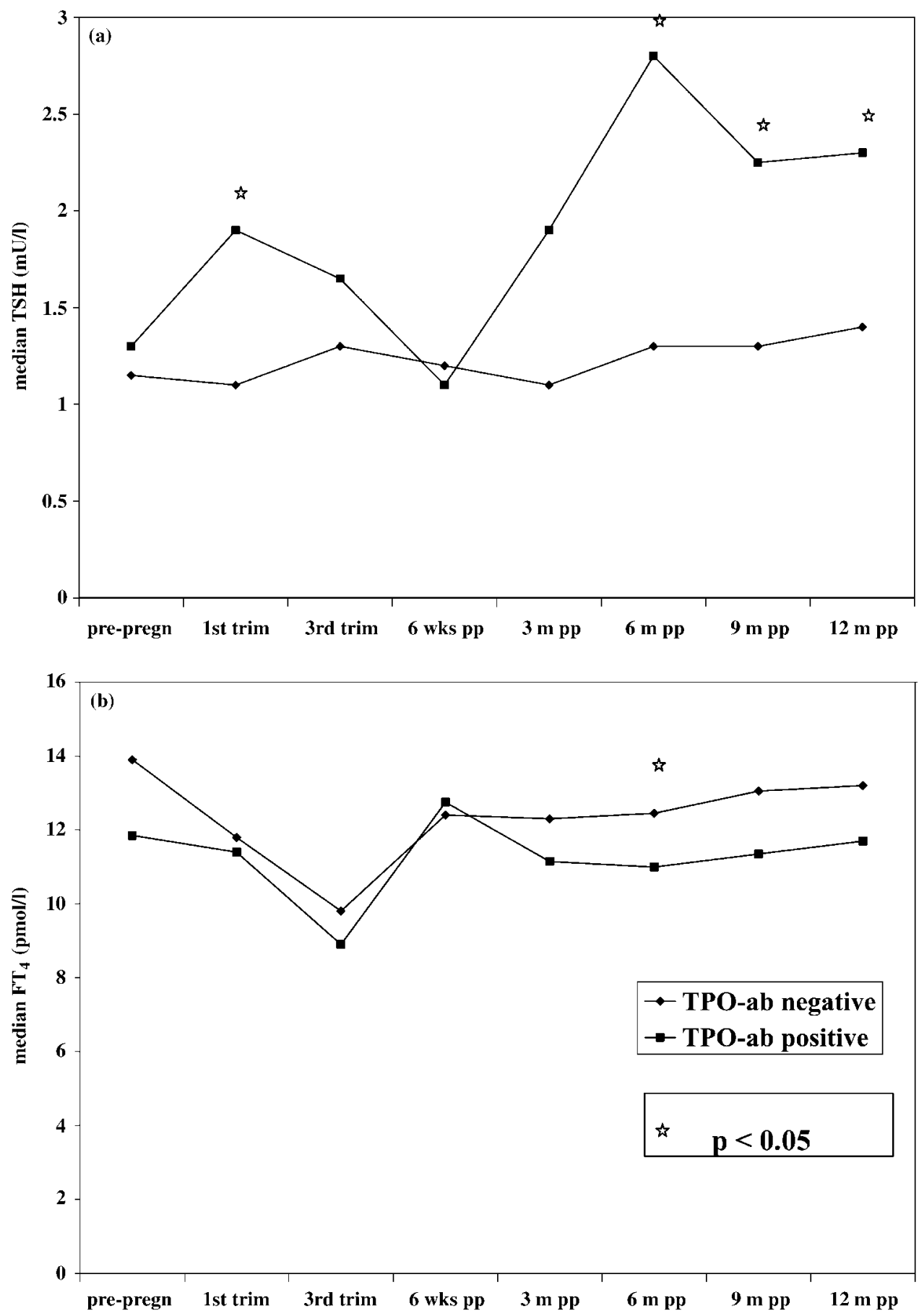

Figure 3 (a) TSH (median) according to TPO-ab status. TSH values in women positive for TPO-ab pre-pregnancy or in the first trimester are higher in the first trimester of pregnancy and at three time points in the postpartum period compared with women without TPO-ab. (b) $\mathrm{FT}_{4}$ (median) according to TPO-ab status. $\mathrm{FT}_{4}$ levels in women positive for TPO-ab pre-pregnancy or in the first trimester are higher at 6 months postpartum compared with women without TPO-ab. pre-pregn, pre-pregnant; trim, trimester; wks, weeks; $\mathrm{m}$, months; pp, postpartum.

first trimester, two $(16.7 \%)$ developed overt PPTD. Changing the definition for TPO-ab positivity to more than $50 \mathrm{kU} / \mathrm{l}$ results in exactly the same percentage: 3 of 18 women $(16.7 \%)$.

\section{Discussion}

As might be expected, thyroid auto-antibody positivity was high in DM1 women before pregnancy. Subclinical 


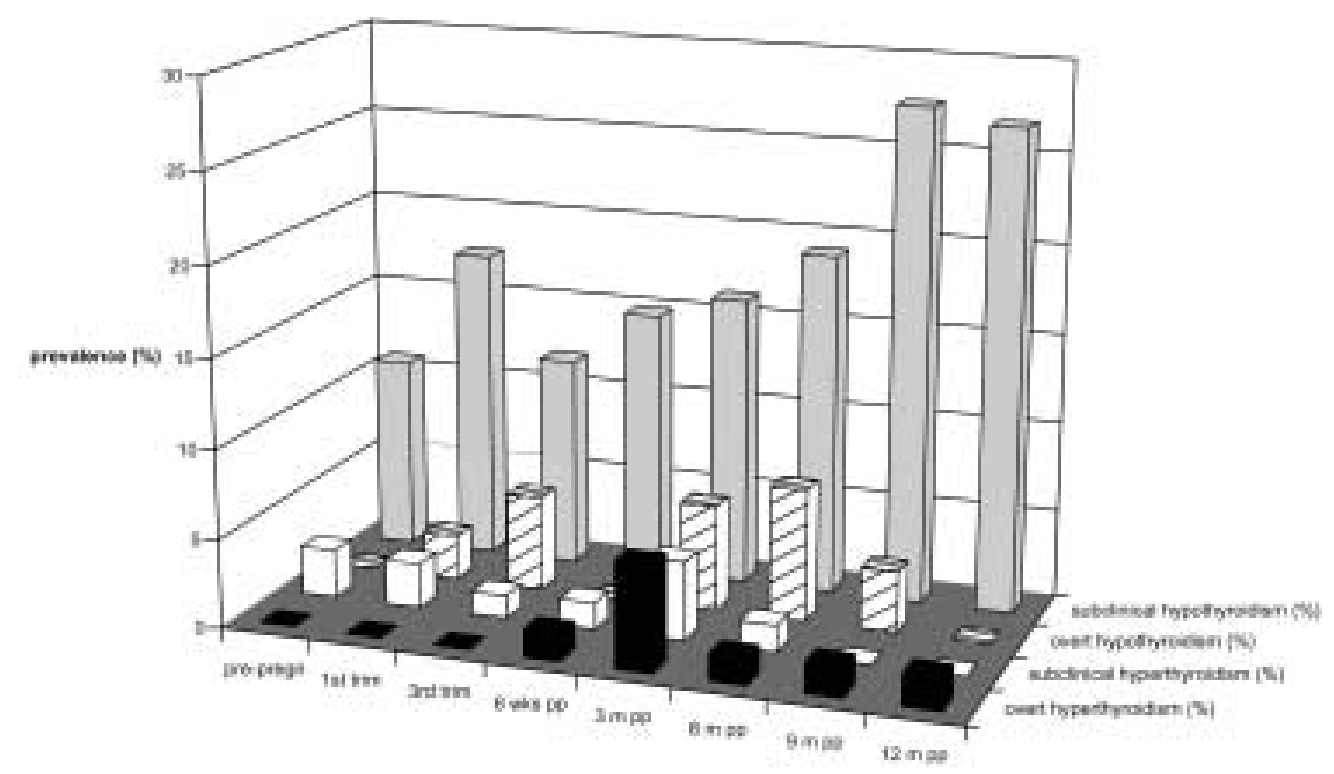

\begin{tabular}{|c|c|c|c|c|c|c|c|c|}
\hline & potanens & Tsime & $30 \mathrm{~mm}$ & $\theta$ Nespos & $3 \pi B$ & $6 m 00$ & $8 m p$ & $2 \approx 60$ \\
\hline 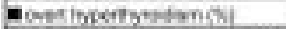 & D & a & 0 & 1.2 & 50 & 15 & $\theta$ & 18 \\
\hline 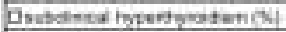 & 27 & 25 & 1.1 & 1.1 & 64 & 28 & 8 & E \\
\hline Bontiosctorsectien is: & D & 25 & $\bar{M}$ & a & 50 & 74 & 34 & t \\
\hline 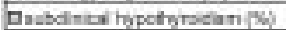 & tive & 775 & 118 & 149 & 162 & $1+1$ & 878 & 289 \\
\hline
\end{tabular}

Figure 4 Prevalence of TD before, during and after pregnancy. The prevalence of subclinical and overt hypothyroidism rises in the postpartum period. Prevalence of subclinical and overt hyperthyroidism shows an increase at 3 months postpartum. pre-pregn, pre-pregnant; trim, trimester; wks, weeks; m, months; pp, postpartum.

hypothyroidism was present in nearly $11 \%$ of the women before pregnancy; no overt hypothyroidism was found at that time point.

As known from previous studies (19) there was a trend for $\mathrm{FT}_{4}$ to decrease during pregnancy and for TSH to gradually rise within normal limits, due to the influence of increasing concentrations of thyroxine binding globuline, the transplacental passage of $\mathrm{FT}_{4}$ and the increased turnover of $\mathrm{FT}_{4}$ by the high activity of placental deiodinase III (8). This trend was enhanced in women with TPO-ab. In these women $\mathrm{FT}_{4}$ reached a subnormal level in the last trimester of pregnancy.

Mild iodine deficiency does not contribute to the fall of $\mathrm{FT}_{4}$ as The Netherlands is an iodine-sufficient country (20). In addition to these normal thyroidal events we assessed a high prevalence of TD during pregnancy (prevalence in first and third trimester 22.5 and $18.4 \%$ respectively). In a previous Dutch study by Kuijpens et al. (17) in the general population the prevalence of TD during gestation was $8 \%$ (23 of 291); consequently, women with DM1 are at nearly three times the risk of having TD in pregnancy vs the normal population. Most of the women in our study had subclinical hypothyroidism. In the general Dutch population more than half of TD concerned a (sub)clinical hyperthyroidism around 12 weeks of pregnancy, which is probably associated with high beta-chorionic gonadotrophin levels at that time (21).
The incidence of TD in diabetic pregnancy was also high: $19.4 \%$ (12.9\% subclinical hypothyroidism).

The high prevalence of an elevated TSH in diabetic women during pregnancy most probably reflects the higher incidence of chronic auto-immune thyroiditis in DM1, which compromises slightly the ability of the maternal thyroid gland to meet the increased demands for thyroid hormone supply during pregnancy. Although the presence of TPO-ab was not related to elevated TSH values in pregnancy, other explanations are less likely, especially because glycaemic control did not differ between patients with or without TPO-ab nor between patients with a normal or elevated TSH. Likewise, the increased incidence of PPTD in DM1 women is most likely related to the overall higher incidence of chronic auto-immune thyroiditis in DM1 and the well-known rebound auto-immune reactions postpartum. Former studies in pregnant women with DM1 revealed a varying prevalence or incidence of TD. Jovanovic-Peterson \& Peterson (10) reported an incidence of overt hypothyroidism in the second trimester of $16 \%$, but from the eight patients developing overt hypothyroidism six already had subclinical hypothyroidism in the first trimester and no data of these patients are given from the pre-pregnant state. Bech et al. (9) did not find any TD during pregnancy in 85 pregnant women with DM1. Alvarez-Marfany et al. (16) found 2 of 41 women (5\%) with DM1 to 
have TD during pregnancy. In a recent study of Fernandez-Soto et al. (22), 3 of 20 women (15\%) developed TD during pregnancy $(\mathrm{TSH}>10 \mathrm{mU} / \mathrm{l})$, but again no data as to the thyroid function before pregnancy are presented.

More than half of the women (51\%) had some form of TD in the first postpartum year (many of them had subclinical hypothyroidism), which is unexpectedly high, but may be partly explained by the low upper level of the TSH range we applied. On the other hand, using the same normal range for TSH, PPTD in the general Dutch population was only $12.4 \%$ (17), so all together there seems to be a 4-fold increase of PPTD in the diabetic women.

The prevalence of overt PPTD in the general population in The Netherlands is $5.2 \%$ (17). The prevalence of overt PPTD in our population was about three times higher $(15.9 \%)$. This 3-fold increase was also seen in three former studies in women with $\operatorname{DM1}(9,15,16)$.

Subclinical hypothyroidism and the presence of TPO-ab in the first trimester are risk factors (positive predictive value 0.36 and 0.35 respectively). When screening for TD in early pregnancy is instituted, most women with detected subclinical hypothyroidism will probably be treated with L-thyroxine. It is uncertain what effect this will have on the occurrence of overt PPTD (23).

The incidence of PPTD (defined as the occurrence of TD in the first postpartum year in women who were euthyroid during pregnancy) was 38\% (compared with $10 \%$ in the general population (17)). Overt PPTD de novo is found in $10 \%$ of the women, which is in accord with the incidence found in Denmark (10.5\% (9)), using approximately the same criteria. Alvarez-Marfany et al. (16) found an incidence of $25 \%$, but their criteria for PPTD were slightly different (TSH $>5$ or $<0.2 \mathrm{mU} / \mathrm{l}$ ) and may have included subclinical thyroid failure as well.

As most patients with overt hypothyroidism have symptoms and may benefit from treatment the figures given for prevalence and incidence of overt TD seem the most clinically relevant.

In accord with the work of Alvarez-Marfany et al. (17) we found no correlation of newly developed PPTD with TPO-ab positivity in pregnancy, neither was there any relationship with TSH level during pregnancy.

In contrast with the findings of Bech et al. (9) and Fernandez-Soto et al. (22) HbA1c during pregnancy in women who were positive for TPO-ab in the pre-pregnancy period or in the first trimester of pregnancy was not significantly higher than the women without TPO-ab (7.08 vs $6.96 \%)$. The same was true when comparing women with and without PPTD.

In conclusion we found a high incidence and prevalence of TD in women with DM1 in pregnancy and in the first postpartum year. TD during pregnancy was observed in $22.5 \%$ (first trimester) and $18.4 \%$ (third trimester), and mostly concerned subclinical hypothyroidism. Baseline characteristics of women with TD in pregnancy did not differ from those without TD. Overt PPTD was seen in 15.9\%. Incidence of PPTD was $10 \%$. Patients with PPTD were slightly older than those without PPTD and prevalence of TPO-ab was higher in these women.

The high prevalence of subclinical TD pre-pregnancy and the high prevalence and incidence of subclinical and overt TD during pregnancy in this study is important in the light of the accumulating evidence that insufficient thyroid hormonal reserve in pregnant women may have deleterious consequences for the psychomotor development of the offspring (24-26). For this reason it has been recommended to screen all women with DM1 for TD before pregnancy, and otherwise as early as possible in pregnancy. When thyroid function pre-pregnancy is normal, the screening should be repeated in the first trimester.

Considering the symptomatology that accompanies PPTD (13) it is advisable to detect PPTD as early as possible. TPO-ab do not have sufficient predictive value for newly developing TD to be of any value. Screening for PPTD can best be done by measuring TSH at 3 and 6 months postpartum.

\section{Acknowledgements}

This study has been made possible by the efforts of the following endocrinologists throughout The Netherlands: Alex Arntzenius (Haarlem), Evert van Ballegooie (Zwolle), Annie Alberda, Jaap van Doormaal, Thera Links (Groningen), Bert Bravenboer (Eindhoven), Rob Heine, Bob Michels, Manon Schreuder (Amsterdam) Jasper Radder (Leiden) and Nel Geelhoed-Duijvestijn (The Hague). Clinical chemist Cas Weijkamp (Winterswijk) provided the conversion factors for the HbA1c measurements. The authors wish to thank them all for their great help. This study was supported by a grant of the Diabetes Fonds Nederland.

\section{References}

1 Riley WJ, Maclaren NK, Lezotte DC, Spillar RP \& Rosenbloom AL. Thyroid autoimmunity in insulin-dependent diabetes mellitus: the case for routine screening. Journal of Pediatrics 198199 350-354.

2 Neufeld M, Maclaren NK, Riley WJ, Lezotte D, McLaughlin JV, Silverstein $\mathrm{J}$ et al. Islet cell and other organ-specific antibodies in US Caucasians and Blacks with insulin-dependent diabetes mellitus. Diabetes 198029 589-592.

3 Betterle C, Zanette F, Pedini B, Presotto F, Rapp LB, Monciotti et al. Clinical and subclinical organ-specific auto-immune manifestations in type 1 (insulin-dependent) diabetic patients and their first degree relatives. Diabetologia 198426 431-436.

4 Gray RS, Borsey DQ, Seth J, Herd R, Brown NS \& Clarke BF. Prevalence of subclinical thyroid failure in insulin-dependent diabetes. Journal of Clinical Endocrinology and Metabolism 1980 50 1034-1037. 
5 Perros P, McCrimmon RJ, Shaw G \& Frier BM. Frequency of thyroid dysfunction in diabetic patients: value of annual screening. Diabetic Medicine $199512622-627$.

6 Glinoer D, Riahi M, Grün JP \& Kinthaert J. Risk of subclinical hypothyroidism in pregnant women with asymptomatic autoimmune thyroid disorders. Journal of Clinical Endocrinology and Metabolism 199479 197-204.

7 Davies TF. The thyroid immunology of the postpartum period. Thyroid $19999675-684$.

8 Glinoer D. What happens to the normal thyroid during pregnancy? Thyroid 1999 $9631-635$.

9 Bech K, Hoier-Madsen M, Feldt-Rasmussen U, Moller-Jensen B, Molsted-Pedersen \& Kühl C. Thyroid function and autoimmune manifestations in insulin-dependent diabetes mellitus during and after pregnancy. Acta Endocrinologica $1991 \mathbf{1 2 4} 534-539$.

10 Jovanovic-Peterson L \& Peterson CM. De novo clinical hypothyroidism in pregnancies complicated by type I diabetes, subclinical hypothyroidism, and proteinuria: a new syndrome. American Journal of Obstetrics and Gynecology 1988159 442-446.

11 Stagnaro-Green A. Postpartum thyroiditis: prevalence, etiology, and clinical implications. Thyroid Today 19934 1-11.

12 Roti E \& degli Uberti E. Post-partum thyroiditis - a clinical update. European Journal of Endocrinology 2002146 275-279.

13 Lazarus JH. Clinical manifestations of postpartum thyroid disease. Thyroid $19999685-689$.

14 Othman S, Phillips DI, Parkes AB, Richards CJ, Harris B, Fung H et al. Long-term follow-up of postpartum thyroiditis. Clinical Endocrinology 199032 559-564.

15 Gerstein HC. Incidence of postpartum thyroid dysfunction in patients with type 1 diabetes mellitus. Annals of Internal Medicine $1993118419-423$

16 Alvarez-Marfany M, Roman SH, Drexler AJ, Robertson C \& Stagnaro-Green A. Long-term prospective study of postpartum thyroid dysfunction in women with insulin dependent diabetes mellitus. Journal of Clinical Endocrinology and Metabolism 1994 79 10-16.

17 Kuijpens JL, Pop VJ, Vader HL, Drexhage HA \& Wiersinga WM. Prediction of post partum TD: can it be improved? European Journal of Endocrinology $199813936-43$.

18 Weijkamp, C. Glycohemoglobin - quality assessment and improvement. PhD Thesis 1995. Groningen University, The Netherlands.
19 Glinoer D, de Nayer P, Bourdoux P, Lemone M, Robyn C, van Steirteghem A et al. Regulation of maternal thyroid during pregnancy. Journal of Clinical Endocrinology and Metabolism 1990 $71276-287$.

20 Wiersinga WM, Podoba J, Srbecky M, van Vessem M, van Beeren HC \& Platvoet-Ter Schiphorst MC. A survey of iodine intake and thyroid volume in Dutch schoolchildren: reference values in an iodine-sufficient area and the effect of puberty. European Journal of Endocrinology $2001 \mathbf{1 4 4} 595-603$.

21 Glinoer D, de Nayer P, Robyn C, Lejeune B, Kinthaert J \& Meuris S. Serum levels of intact human chorionic gonadotropin (HCG) and its free alpha and beta subunits, in relation to maternal thyroid stimulation during normal pregnancy. Journal of Endocrinological Investigation $199316881-888$.

22 Fernandez-Soto L, Gonzalez A, Lobon JG, Lopez JA, Peterson CM \& Escobar-Jimenez F. Thyroid peroxidase autoantibodies predict poor metabolic control and need for thyroid treatment in pregnant IDDM women. Diabetes Care 199720 1524-1528.

23 Kämpe O, Jansson R \& Karlson FA. Effects of L-thyroxine and iodide on the development of autoimmune postpartum thyroiditis. Journal of Clinical Endocrinology and Metabolism $1990 \quad \mathbf{7 0}$ 1014-1018.

24 Pop VJ, Kuijpens JL, van Baar AL, Verkerk G, van Son MM, de Vijlder JJ et al. Low maternal free thyroxine concentrations during early pregnancy are associated with impaired psychomotor development in infancy. Clinical Endocrinology 199950 $149-155$.

25 Haddow JE, Palomaki GE, Allan WC, Williams JR, Knight GJ, Gagnon $\mathrm{J}$ et al. Maternal thyroid deficiency during pregnancy and subsequent neuropsychological development of the child. New England Journal of Medicine $1999341549-555$.

26 Muller AF, Drexhage HA \& Berghout A. Postpartum thyroiditis and autoimmune thyroiditis in women of childbearing age: recent insights and consequences for antenatal and postnatal care. Endocrine Reviews 200122 605-630.

Received 11 March 2002

Accepted 10 June 2002 